\title{
Antibody response against Trichinella spiralis in experimentally infected rats is dose dependent
}

\author{
Frits FJ Franssen ${ }^{1 *}$, Manoj Fonville, Katsuhisa Takumi ${ }^{1}$, Isabelle Vallée ${ }^{2}$, Aurélie Grasset ${ }^{2}$, Marie A Koedam , \\ Piet W Wester ${ }^{1}$, Pascal Boireau ${ }^{2}$ and Joke WB van der Giessen ${ }^{1}$
}

\begin{abstract}
Domestic pigs are the main representatives of the domestic cycle of Trichinella spiralis that play a role in transmission to humans. In Europe, backyard pigs of small household farms are the most important risks for humans to obtain trichinellosis. Rats might play a role in the transmission of Trichinella spiralis from domestic to sylvatic animals and vice versa. In order to be able to investigate the role of wild rats in the epidemiology of $T$. spiralis in The Netherlands, we studied the dynamics of antibody response after T. spiralis infections in experimental rats, using infection doses ranging from very low (10 muscle larvae, ML, per rat) to very high (16 $000 \mathrm{ML}$ per rat). To evaluate the feasibility of rats surviving high infection doses with T. spiralis, clinical and pathological parameters were quantified. Serological tools for detecting $T$. spiralis in rats were developed to quantitatively study the correlation between parasite load and immunological response. The results show that an infection dose-dependent antibody response was developed in rats after infection with as low as $10 \mathrm{ML}$ up to a level of $10000 \mathrm{ML}$. A positive correlation was found between the number of recovered ML and serum antibody levels, although specific measured antibody levels correspond to a wide range of LPG values. Serum antibodies of rats that were infected even with 10 or $25 \mathrm{ML}$ could readily be detected by use of the T. spiralis western blot 2 weeks post infection. We conclude that based on these low infection doses, serologic tests are a useful tool to survey T. spiralis in wild rats.
\end{abstract}

\section{Introduction}

Trichinella spiralis is the only known Trichinella species out of 12 recognized species or genotypes [1] that is transmitted and maintained in both a domestic and sylvatic cycle. The T. spiralis sylvatic cycle involves omnivores like the wild boar, carnivores like the wolf and fox, but also scavenger wild rodents $[2,3]$. T. spiralis is distributed worldwide and maintained in pigs as one of the most important representatives of the domestic cycle. In Europe, free ranging pigs of small household farms are the most important risk for public health [3].

Rats play a role in the transmission of $T$. spiralis from domestic to sylvatic animals and vice versa. It has been shown that pigs exposed to rats were infected more often, whereas pigs that were physically separated from rats remained free of Trichinella [4]. Rats in the vicinity of pig farms were infected only when $T$. spiralis occurred in pigs on those farms under low sanitation

\footnotetext{
* Correspondence: frits.franssen@rivm.nl

'Laboratory for Zoonoses and Environmental Microbiology, National Institute for Public Health and the Environment (RIVM), Bilthoven, The Netherlands Full list of author information is available at the end of the article
}

levels $[5,6]$. However, it has been shown that even in the absence of a known source of infection on farm level, $T$. spiralis is able to persist in rats [5].

In the geographical spread and maintenance of $T$. spiralis in nature, humans play a major role. Disposal of infected carcasses of pigs or hunted wild boars, wolves and foxes in nature or on waste disposal sites might be a driving force in spreading $T$. spiralis infections in wild rat populations $[7,8]$. Circumstantial evidence has indicated that an outbreak of $T$. spiralis in outdoor farmed wild boar could be attributed to an invasion of rats from an improperly closed down landfill in the vicinity of the farm [9]. Jovic et al. [10] showed by bioassay using rats, that $T$. spiralis larvae in artificially infected pork meat that had been buried in the ground at a depth of $30-100 \mathrm{~cm}$, remains infective for rats for more than 91 days.

Rats were shown to be a potential reservoir host species of Trichinella using mathematical models, provided that cannibalism occurs [11]. It was argued in that study that rats should be included in the minimal set of wildlife species that maintain the cycle of $T$. spiralis. Even if

\section{() Biomed Central}


rats do not represent an important route of Trichinella distribution, but are merely sentinel species, it might be useful to monitor rats for Trichinella in a wildlife monitoring programme. Wildlife monitoring is one of the tools indicated by the EU regulation 2075/2005EU to control Trichinella [12]. The results of a rodent monitoring might give additional information about Trichinella dynamics in wildlife and might also be useful in a more generic wildlife monitoring programme.

In this study, we developed serological tools to quantitatively study the correlation between parasite load and immunological response of artificially $T$. spiralis infected rats at different infection levels. To augment the dynamics of $T$. spiralis in infected rats using different infection doses, and to evaluate the probability of rats surviving high infection doses with T.spiralis, clinical and pathological parameters are quantitatively described as well.

\section{Materials and methods \\ Experimental infection}

Male Wistar Unilever rats weighing 230-280 g were infected with $T$. spiralis muscle larvae (strain ISS 14), which had been isolated by pepsin- $\mathrm{HCl}$ digestion from previously infected mice or rats. To assess low dose infection, thirty-six rats were divided into six groups of six animals per group and individual rats were infected with 10, 25, 50, 100, 200 and $400 \mathrm{ML}$ per group. To assess high dose infection, an additional thirty rats were divided into ten groups of three animals per group and individual rats were infected with 200, 400, 2 000, 4 000, 6 000, 8 000, 10 000, 12 000, 14000 and 16000 ML per group. On the first day of the experiments, all rats received ML which were delivered directly into the stomach through a gastric tube. Doses of 10 to $400 \mathrm{ML}$ were prepared by counting individual larvae. Doses between 2000 and $16000 \mathrm{ML}$ were prepared by a series of dilutions. At each dilution step, the suspensions were mixed continuously by magnetic stirring to avoid possible sedimentation of the muscle larvae. Blood from rats of both the low and high dose experiments was collected weekly via orbital puncture and sera were stored at $-20^{\circ} \mathrm{C}$ until further use. Rats in the groups of 10000 ML or higher showed signs of severe clinical illness and their diets were changed to soft food to prevent premature termination. Forty-two days post infection (dpi) the rats were euthanized. Body weights and clinical scores were determined daily. Clinical scores were defined as follows: 0-no signs, 1-rough coat and light diarrhea, 2rough coat, animal inactive and severe diarrhea, 3-rough coat, inactivity, severe diarrhea and weight loss, 4-rough coat, inactivity, severe diarrhea, more than $20 \%$ weight loss and decrease in body temperature, 5-death. The experimental protocol was approved by the RIVM Animal Experiments Committee.

\section{Pathology}

At terminal sacrifice at $42 \mathrm{dpi}$, samples of front leg muscle (1 animal per group, highest level of recovered muscle larvae), duodenum, heart, liver, thymus, spleen and mandibular lymph node were collected and fixed in $4 \%$ buffered formaldehyde. Tissues were processed to paraffin blocks and $4 \mu \mathrm{m}$ sections were cut and stained with $H \& E$. All organs were examined in the lowest and highest infection dose groups, and affected organs (spleen, thymus, lymph node and muscle) also from intermediate groups.

At day 8 post infection (pi), an autopsy was performed on the animals of the highest dose group (16 $000 \mathrm{ML})$, of which the organs were examined as described above. In addition, lungs and three muscles (diaphragm, masseter and quadriceps femoris muscles) and the intestines were examined histologically (small intestine-4 locations, large intestine-3 locations). Selected organs were stained with PAS additional to $\mathrm{H} \& \mathrm{E}$.

\section{Isolation of muscle larvae}

From each rat, the muscle tissue was separated and $T$. spiralis ML were isolated by artificial digestion according to EU regulation 2075/2005 [12]. Briefly, after weighing the carcass, all muscle tissue from each rat was isolated, its weight was established and it was digested using pepsin- $\mathrm{HCl}$ for $30 \mathrm{~min}$ at $45 \pm 1^{\circ} \mathrm{C}$. After two successive sedimentation steps, the resulting suspension containing ML was transferred to Petri dishes and the number of isolated larvae was determined by two technicians. These counts were used to calculate average values and the number of larvae per gram (LPG) muscle tissue per rat.

\section{Production of Trichinella ES antigen}

T. spiralis excretory/secretory (E/S) antigen was produced as described previously by Gamble [13]. Briefly, Trichinella ML (ISS 14) were isolated from mouse muscle tissue by artificial digestion and washed three times in PBS containing penicillin (12 mg per $100 \mathrm{~mL}$ ) and streptomycin $(20 \mathrm{mg})$. Washed ML were incubated at a concentration of 10000 larvae per $\mathrm{mL}$ in a $\mathrm{CO}_{2}$ incubator for $18 \mathrm{~h}$ at $37^{\circ} \mathrm{C}$ in $250 \mathrm{~cm}^{2}$ culture flasks containing $70 \mathrm{~mL}$ RPMI 1640 culture medium (supplemented with $1 \%$ glutamine and $1 \%$ penicillin/streptomycin, Gibco 10378, Invitrogen, Bleiswijk, The Netherlands). The culture medium was separated from the ML by centrifugation and the supernatant was concentrated in a dialysis membrane (Spectra/Por molecularporous membrane MWCO 6-8 000, Spectrum Laboratories, Inc., Rancho Dominguez, USA) by dehydration using polyethylene glycol 20000 (PEG, Fluka 81300, Sigma-Aldrich Chemie GMBH, Steinheim, Germany). The concentrated culture medium was dialyzed two times over-night against PBS 
at $4^{\circ} \mathrm{C}$ and the dialyzed and concentrated solution containing $T$. spiralis ES proteins was further concentrated using $5 \mathrm{kD}$ molecular weight cut-off filters (Amicon Ultra Centrifugal Filter Devices, Millipore, Carrigtwohill, Ireland). The protein concentration was determined by BCA protein assay (Pierce, Rockford, IL, USA).

\section{In-house ELISA}

Antibody responses of the infected rats were determined by an in-house ELISA. Flat-bottom ELISA plates (Nunc 66904) were coated with $0.125 \mu \mathrm{g}$ of $T$. spiralis ES antigen per well for one hour at $37^{\circ} \mathrm{C}$. After washing (PBS/ $0.05 \%$ Tween 20 (PBS-T)) and saturation (1\% BSA in PBS-T at $4^{\circ} \mathrm{C}$ overnight), sera were applied $(100 \mu \mathrm{L}$ per well, $1 / 100$ dilution in PBS-T) for one hour at $37^{\circ} \mathrm{C}$. After washing, each well was incubated for one hour at $37^{\circ} \mathrm{C}$ with $100 \mu \mathrm{L}$ Goat anti-Rat IgG conjugated to Horse Radish Peroxidase (1/8000 in $1 \%$ BSA/PBS-T). After washing, antigen-antibody interaction was visualized by adding $100 \mu \mathrm{L}$ Sure Blue ${ }^{\mathrm{TM}}$ TMB substrate solution (KPL, Gaithersburg, USA) per well and incubation for ten minutes at room temperature. The reaction was stopped with $100 \mu \mathrm{L} 2 \mathrm{M} \mathrm{H}_{2} \mathrm{SO}_{4}$ and optical density (OD) values were determined with an ELISA reader (Bio-Tek EL808 Ultra Microplate Reader, Bio-Tek Instruments, Inc.) at $450 \mathrm{~nm}$.

\section{Calculation of normalized OD and cut-off}

Sera were tested in duplicate and normalized OD minus blank $\left(O D_{n}\right)$ values were calculated using the formula $\mathrm{OD}_{\mathrm{n}}=(\mathrm{OD}-b) / a$, where $\mathrm{OD}$ is the average OD minus blank value of the duplicates, $a$ is the $\mathrm{x}$-variable and $b$ the intercept from the linear regression analysis tool in Microsoft Excel. Positive and negative rat control sera were used to determine variables $a$ and $b$ for each ELISA plate to correct for inter-plate and day-to-day variation. The $\mathrm{OD}_{\mathrm{n}}$ values of pre-immune (day 0) sera of all experimental rats was used to define a cut-off level as average $\mathrm{OD}_{\mathrm{n}}$ plus 2 times standard deviation.

\section{Western blot}

T.spiralis ES antigen ( $1 \mu$ g total protein per lane) was separated by SDS-Page and transferred to nitrocellulose membrane (Trans-Blot ${ }^{\circledR}$ Transfer Medium, Bio-Rad Laboratories, Hercules, CA, USA). After saturation of the membrane with $1 \% \mathrm{BSA}$ in PBS-T (BSA/PBS-T), serum dilutions (1:50 or 1:300 (positive control) in BSA/ PBS-T) were applied and immunoreactions were visualized with Goat anti Rat IgG conjugated to Horse Radish Peroxidase (Sigma-Aldrich, St. Louis, USA, 1:5000 in BSA/PBS-T) and ECL Detection Reagent (GE Healthcare Ltd, Little Chalfont, Buckinghamshire, UK), followed by detection on a Luminescent Image Analyzer (LAS-3000, Fuji Photo Film Co., Ltd, Tokyo, Japan).
Rats infected with 10,25 or $50 \mathrm{ML}$ with $\mathrm{OD}_{\mathrm{n}}$ values roughly equal to the average $\mathrm{OD}_{\mathrm{n}}$ in the T.spiralis ES ELISA were selected for testing in the western blot.

\section{Statistical analysis}

The correlation between measured $\mathrm{OD}_{\mathrm{n}}$ values and ${ }^{10} \log$ transformed values of recovered larvae per gram (LPG) were determined using Microsoft Excel statistical section. From the 2D normal distribution for $\mathrm{OD}_{\mathrm{n}}$ and LPG, low estimate ( 0.05 percentile) and high estimate (0.95 percentile) of the conditional distribution of LPG given the $\mathrm{OD}_{\mathrm{n}}$ value was determined.

For analysis of the dose effect on $\mathrm{OD}_{\mathrm{n}}$ per time point, the Generalized Linear Model (GLM) Fit of Mathematica 8.0.1.0 (Wolfram, Inc, Champaing IL, USA) was used. The combined effect of time and dose was calculated in the GLM using the formula $\mathrm{OD}_{\mathrm{n}}=a+b \cdot$ dose-day. Probability levels less than 0.05 were considered significant.

\section{Results}

\section{Clinical scores and body weight}

No clinical signs were observed in animals that were infected with less than $6000 \mathrm{ML}$. The rats that were infected with 6000 or $8000 \mathrm{ML}$ showed mild clinical symptoms (maximum average clinical score 1-1.25 on day 8 pi) from day 5 to day 10 pi. In animals that were infected with 10 000-14 $000 \mathrm{ML}$, clinical symptoms started earlier (day 2 pi), lasted longer (day 18 pi) and the maximum average clinical score reached level 3 in these animals (Figure 1). The animals in the highest dose group (16 $000 \mathrm{ML}$ ) were severely affected at day 8 pi (clinical score level 4, body weight loss $21 \pm 2.6 \%$, Figure 1) and it was decided to euthanize these animals at day 8 pi.

The average bodyweight of rats infected with 200-2000 ML showed no decrease after infection with $T$. spiralis ML. The animals in the higher infection dose groups lost bodyweight in a dose dependent manner [11]. Briefly, between day 5 and day 8 pi, the average bodyweight of the rats infected with $4000 \mathrm{ML}$ decreased with $7.2 \% \pm 5.5 \%$. Rats infected with $6000-14000 \mathrm{ML}$ lost $15.8 \% \pm 4.3 \%$ bodyweight. From day 10 onwards, the rats of the $4000-14000 \mathrm{ML}$ infection groups regained bodyweight $(129 \mathrm{~g} \pm 24 \mathrm{~g}$ from $8 \mathrm{dpi}$ to 42 dpi) at the same rate as the animals in the 200-2 000 ML groups (123 $\mathrm{g} \pm 4 \mathrm{~g})$.

\section{Pathology}

The rats that received $16000 \mathrm{ML}$ were euthanized at day 8 pi and were necropsied immediately after euthanasia. All three animals showed hypermotility of the pale intestine. The duodenum up to the ascending colon was distended with clear, slightly viscous fluid containing 


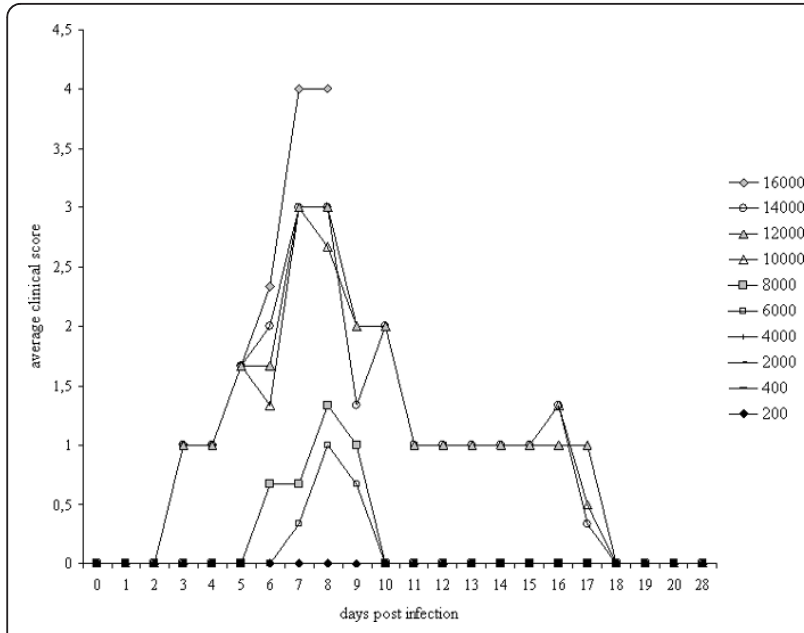

Figure 1 Average clinical scores per dose group $(n=3)$. Clinical signs developed in animals that were infected with $6000 \mathrm{ML}$ or more, mainly during the intestinal phase of Trichinella. In animals that were infected with $10000-16000 \mathrm{ML}$, clinical symptoms set on earlier and lasted longer as compared to rats that received lower doses.

minute flakes. Microscopically, shed villus tips were visible in these contents. The stomach contained only bile. Peyers patches and mesenteric lymph nodes were markedly enlarged. The spleen was small and the thymus very small. No body fat was present.

Histopathologically, intestinal lesions were most severe in the proximal small intestine and caecum. Trichinella females were most numerous in the proximal small intestine but present up to the descending colon. The mucosa of the duodenum and jejunum was covered with a thick layer of PAS-positive mucus containing extruded epithelial cells. Erosion was minimal due to obvious efficient fusion of the remaining short and distorted villi (Figure 2). Immaturity of the superficial epithelium was generally observed. Inflammatory infiltration in the lamina propria consisted mainly of eosinophilic leucocytes, many of which were degranulated. In the caecum, the submucosa was infiltrated and oedematous. Plasmacytosis of the medullary cords was responsible for the enlargement of the mesenteric lymph nodes. Other lymph nodes showed lymphoid atrophy (Figure 3a). Splenic peri-arteriolar lymphocyte sheath (PALS) and marginal zones were atrophic (Figure 3c). Follicle centres in the spleen and lymph nodes showed slight necrosis. In the thymus cortex, there was complete loss of lymphocytes (Figure 3e). No abnormalities were found in the liver, heart and lung. In the muscles, notably in the diaphragm, acute degeneration of myocytes was seen, together with some newborn larvae.

At terminal sacrifice of the other animals on day 42 pi, no abnormalities in the heart, liver and duodenum

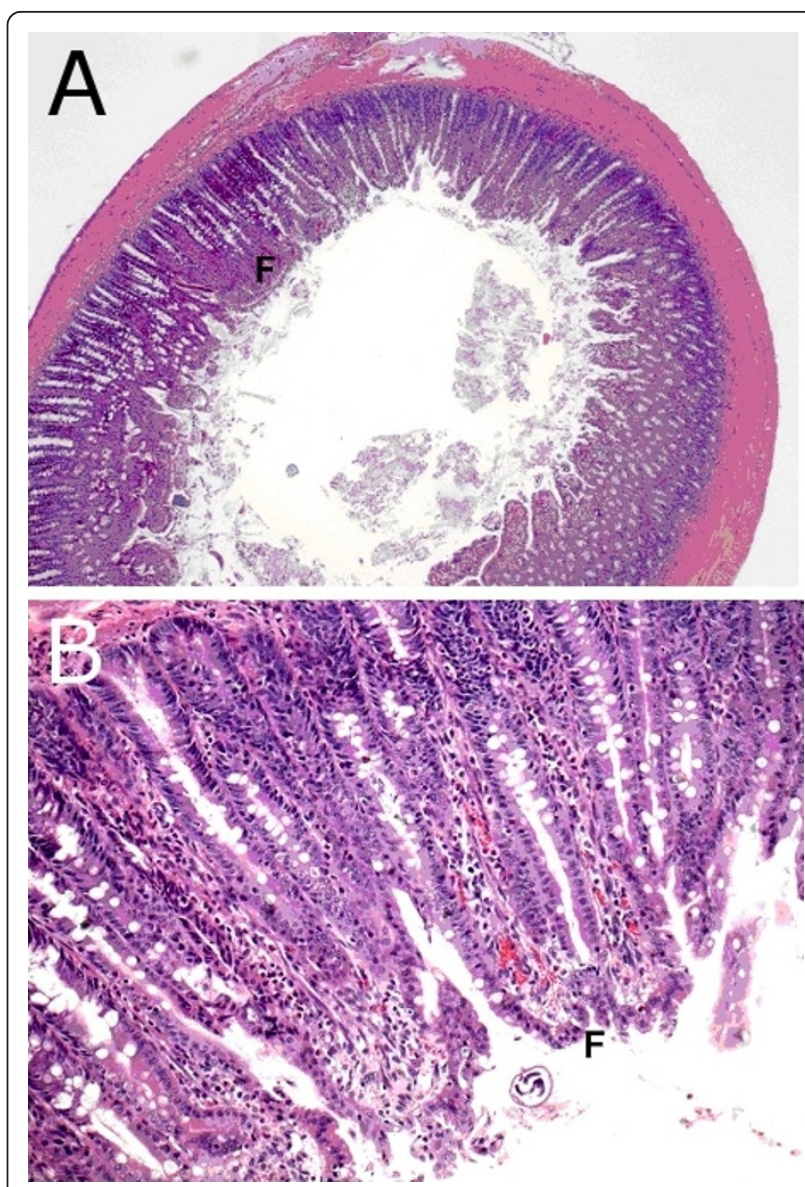

Figure 2 A. Histopathology of duodenum at $8 \mathrm{dpi}(16000 \mathrm{ML}$, H\&E). A. Villi are fused (F) and contain small hemorrhages as seen at magnification 20x. B. detail from A at magnification 100X.

were found in any of the infection groups. The cellularity in the lamina propria of the duodenum was prominent but no difference between the 200 and the 14000 ML dose groups was observed. Mandibular lymph nodes showed an activated appearance as seen by abundant secondary follicles, paracortex and medullary cords (Figure $3 \mathrm{~b}$ ). The spleen was often congested with prominent marginal zones (Figure 3d). The thymus was well developed and showed no signs of regression (Figure 3f). Only a single muscle sample was taken per treatment group. The most prominent changes were seen in the highest dose groups. Individual myofibers were swollen and degenerated and contained pale amorphous material harboring larvae (nurse cells). Muscle fibers in the vicinity were often degenerated. Inflammatory cells (predominantly macrophages) were abundantly surrounding affected fibers, but typically not directly in or on the nurse cells (the larvae or capsules) (Figure 4).

No difference was found in lymphoid organs from the intermediate dose groups. The numbers of muscle larvae increased with infection dose as shown after counting, 


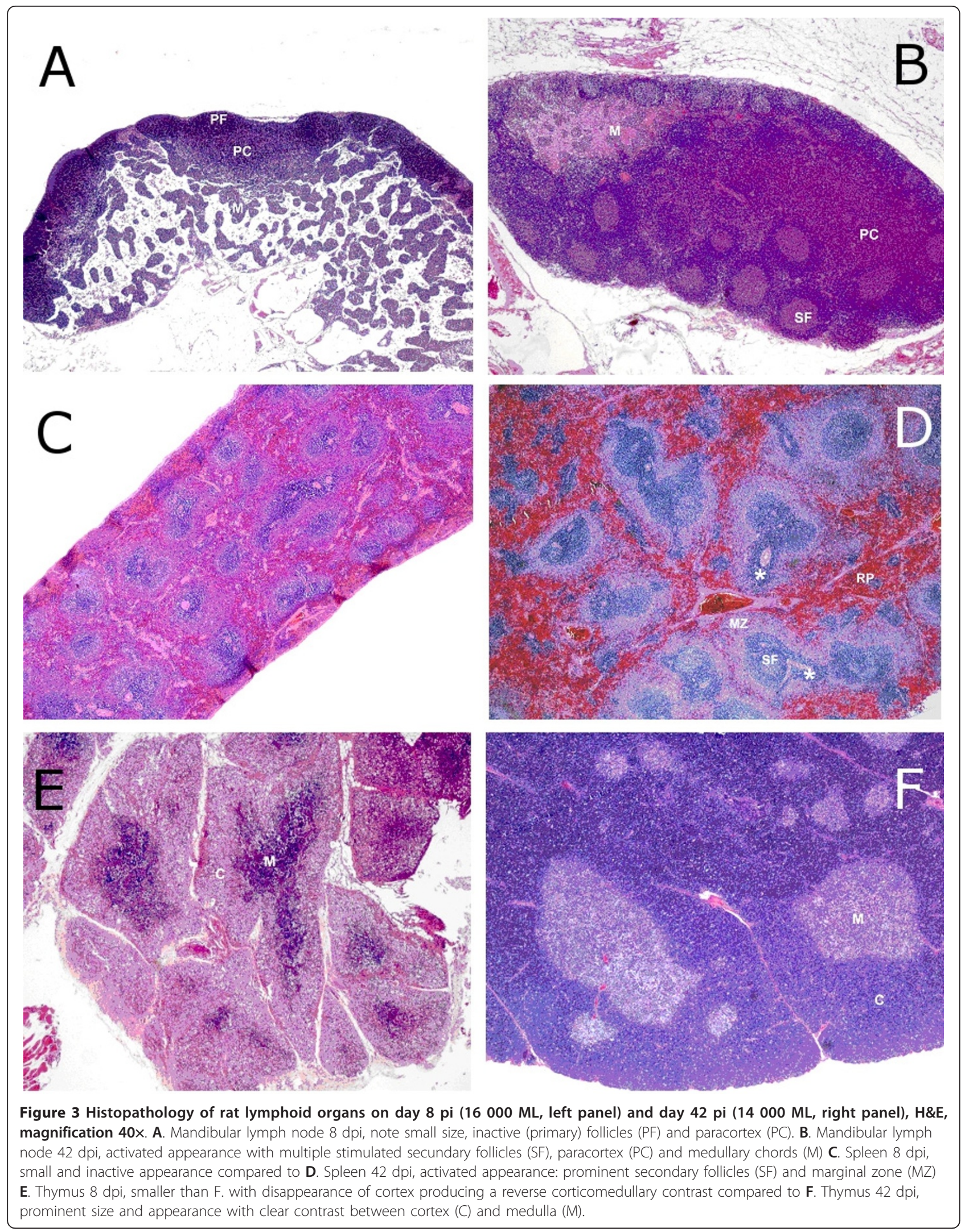




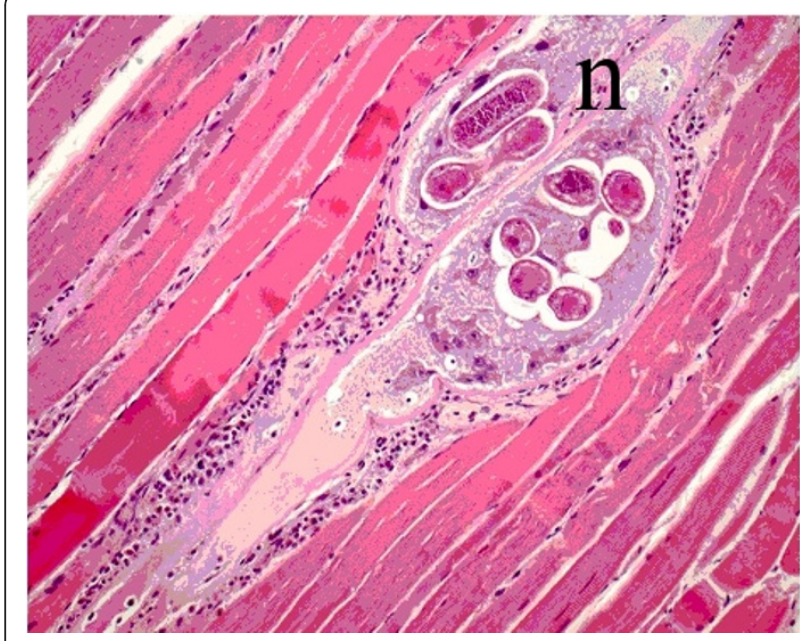

Figure 4 Histopathology of muscle tissue at 42 dpi (14 $000 \mathrm{ML}$, H\&E, magnification 200x). Swollen transformed muscle fibers contain live muscle larvae (Nurse cells, n) surrounded by inflammatory cells (mainly macrophages).

but these data were of limited quantitative value, as only 1 muscle sample per treatment group was taken, and significant variation per muscle sample is to be expected.

\section{Recovery of muscle larvae}

Administration of $10 \mathrm{~T}$. spiralis ML resulted in active infections in all animals from which on average $6.96 \pm$ 4.42 larvae per gram (LPG) could be recovered. Increasing infection doses resulted in increasing numbers of recovered larvae, in a non-linear manner as was demonstrated in our laboratory [11]. Infection doses above 10 $000 \mathrm{ML}$ resulted in decreasing numbers of recovered muscle larvae at day 42 pi. No ML could be recovered on day 8 pi from muscle tissue of animals infected with $16000 \mathrm{ML}$.

\section{Correlation between OD and LPG}

All experimentally infected rats seroconverted between 7 and 14 dpi with OD levels well above cut-off, except for the animals of the $10 \mathrm{ML}$ infection group, which seroconverted between 14 and $21 \mathrm{dpi}$. In general, there is a positive correlation between $\mathrm{OD}_{\mathrm{n}} \pm \mathrm{SE}$ with infection dose, although there is a remarkable difference between low (10-400 ML) and high (2 000-10 $000 \mathrm{ML}$ ) infection dose ranges (Figure $5 \mathrm{a}) . \mathrm{OD}_{\mathrm{n}}$ values of rats that were infected with 12000 and $14000 \mathrm{ML}$ declined as compared to the $10000 \mathrm{ML}$ infection group, reflecting the decline in LPG yield at $42 \mathrm{dpi}$ in these infection groups (data not shown). There was a significant positive correlation between $\mathrm{OD}_{\mathrm{n}}$ values and infection dose in the range $2000-10000 \mathrm{ML}$ at all time points $(P<0.05$, Figure $5 \mathrm{~b})$. In the lower dose groups there was a significant positive correlation at all time points, except $7 \mathrm{dpi}(P<$ 0.05 , Figure $5 \mathrm{c}$ ). The increase of $\mathrm{OD}_{\mathrm{n}}$ values over time was significant for all infection groups $(P<0.05)$.

$\mathrm{OD}_{\mathrm{n}}$ values of individual animals measured at $42 \mathrm{dpi}$ plotted against ${ }^{10} \mathrm{log}$ recovered LPG yielded a positive correlation $\left(R^{2}=0.668\right.$, Figure 6$)$. However, a wide range of LPG values corresponds to a specific $O D_{n}$ value (Table 1 ).

\section{Western blot analysis}

Infection with as little as $10 \mathrm{ML}$ is sufficient to generate an IgG response on $14 \mathrm{dpi}$ in the $T$. spiralis ES western blot. Although the signal was fairly weak with a serum dilution of 1:50, bands were clearly visible (Figure 7 ). Moreover, by using Western blot assay, seroconversion was detected at $14 \mathrm{dpi}$, which was one week earlier for this low infection level, as compared to ELISA. The seroconversion between day 14 and 21 pi as measured with ELISA was confirmed by an increase in signal with Western blot. With increasing infection dose, the number and intensity of recognized bands is enhanced.

\section{Discussion}

The present study confirmed that $T$. spiralis is highly infective at very low doses in Wistar rats and can easily be detected by serology or pathogen detection using digestion. We describe the dynamics of $T$. spiralis infection in rats using doses ranging from very low (10 ML per rat) to very high $(16000 \mathrm{ML})$, with the aim of studying the usefulness of rats as sentinel animals in a wildlife monitoring program for Trichinella. Rats are sentinels for several human pathogens and have several advantages for epidemiological studies as mentioned by Psaroulaki et al. [14]. This is the first report describing the clinical, pathological, immunological and parasitological findings using a dose range of $T$. spiralis in experimental infection in rats this wide.

Infection with 10-2 $000 \mathrm{ML}$ caused no clinical symptoms in experimentally infected rats. Body weights of rats infected with $4000 \mathrm{ML}$ or more, declined in a dose-dependent manner until day 8-9 pi. This was probably caused by diarrhea and a lesser food intake during the intestinal phase of $T$. spiralis infection as observed before by Aulí and Fernández [15]. Although the probability of survival might have been biased by administering soft food, all animals, except the rats in the highest dose group (16 $000 \mathrm{ML}$ ) were able to overcome illness, regained weight and survived until the end of the experiment.

This was corroborated by the differences in post mortem pathological findings between rats euthanized and examined at day 8 pi (16 $000 \mathrm{ML}$ infection group) and rats euthanized at day 42 (200-14 $000 \mathrm{ML})$. During the intestinal phase of $T$. spiralis (day 8 pi, $16000 \mathrm{ML}$ ), rats 

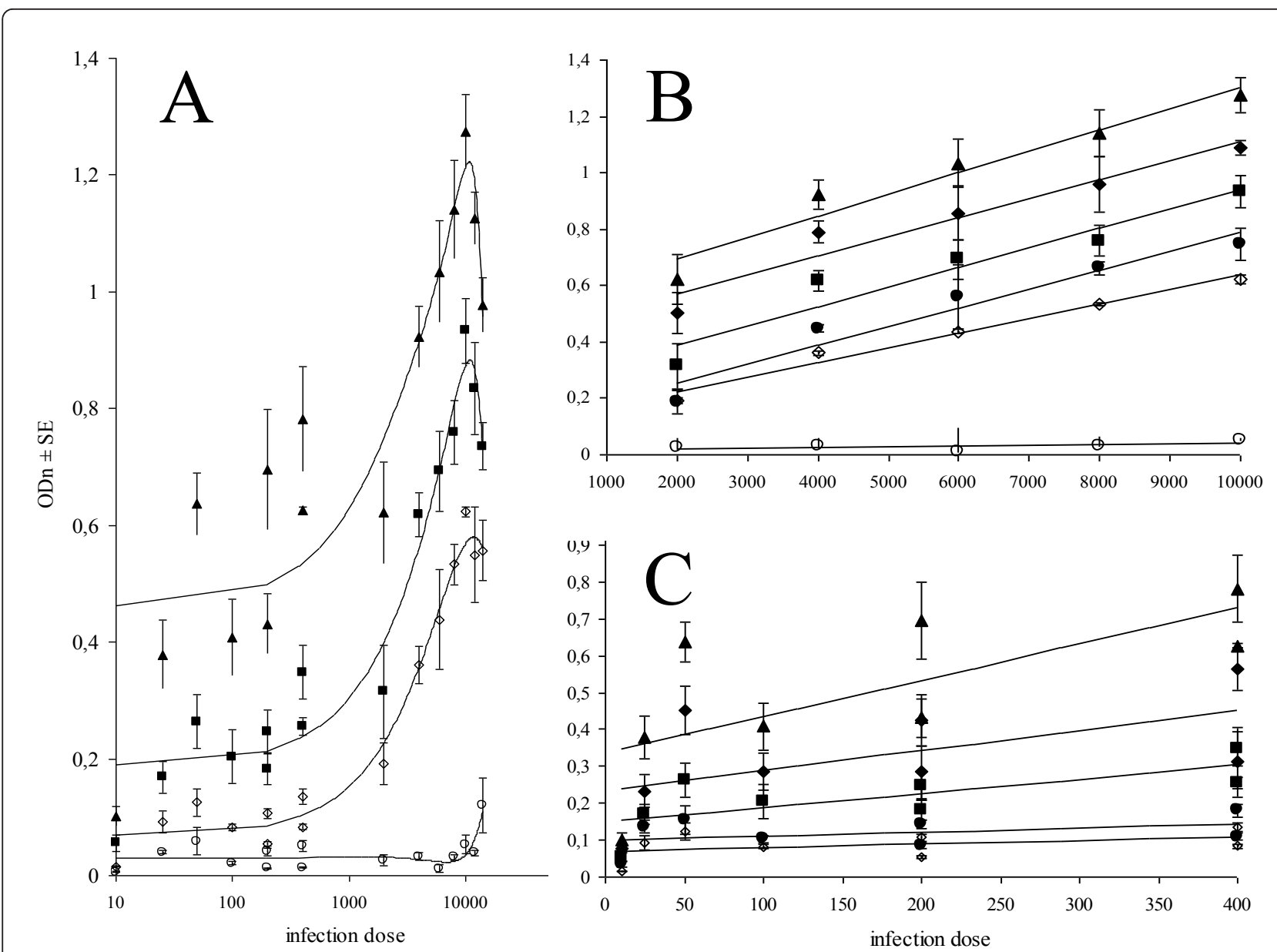

Figure 5 Measured ODn values plotted against infection dose. Immunological response on day 7 (open circle), day 14 (open diamond), day 21 (black circle), day 28 (black square), day 35 (black diamond) and day 42 (black triangle) pi. Infection doses 200 and 400 ML of both low and high dose experiments are displayed. A. Average $\mathrm{OD}_{n} \pm \mathrm{SE}$ values show a positive correlation with infection, both in time and infection dose, although less pronounced at low infection doses (10-400 ML). At the highest doses (12 000-14 $000 \mathrm{ML}), \mathrm{OD}_{\mathrm{n}}$ values decline between day 14 and day 42 pi as compared to the $10000 \mathrm{ML}$ infection group. Intermediate time points are not shown for the sake of clarity. $\mathbf{B}$. Average $O D_{n}$ values of infection groups $2000-10000 \mathrm{ML}$ correlate significantly $(P<0.05)$ with infection dose on all time points $\mathbf{C}$. Average $\mathrm{OD}_{\mathrm{n}}$ values of infection groups 10-400 ML correlate significantly $(P<0.05)$ with infection dose on all time points, except $7 \mathrm{dpi}$.

showed increased mucus production and intestinal hypermotility. This was in accordance with the model of Khan and Collins [16], which describes enteritis induced Th2 immune response resulting in intestinal muscle hyperactivity and increased mucus production by goblet cells.

Histological changes were most prominent in the duodenum at day $8 \mathrm{pi}$, while on day 42 the duodenum was not altered in any of the other infection groups. Also the thymus showed severe atrophy at day $8 \mathrm{pi}$, but showed no pathological alterations after $42 \mathrm{dpi}$ in the other infection groups. Both features indicate successful regeneration of these organs after regression and inactivity indicative of severe biological stress. Findings in the spleen and (mandibular) lymph nodes seen on day 42 pi were considered indicative of systemic immune stimulation. The finding of wild caught Rattus norvegicus with very high LPG, ranging from 5720 to 7692 $[5,7]$, indicates that also in nature wild rats are able to survive these high infection levels.

Infection with high doses of $T$. spiralis ML was characterized by a large variability in the numbers of muscle larvae that developed in individual rats. The maximum number of recovered muscle larvae was found in rats infected with $10000 \mathrm{ML}$. Rats infected with $12000 \mathrm{ML}$ developed fewer muscle larvae and infection with 14 $000 \mathrm{ML}$ produced the fewest muscle larvae. This was probably due to severe competition for space (in the intestinal epithelium as well as in muscle fibers) and nutrients, in combination with expulsion of $T$. spiralis intestinal stages by the host. No ML could be recovered on day 8 pi from the muscle tissue of animals infected 


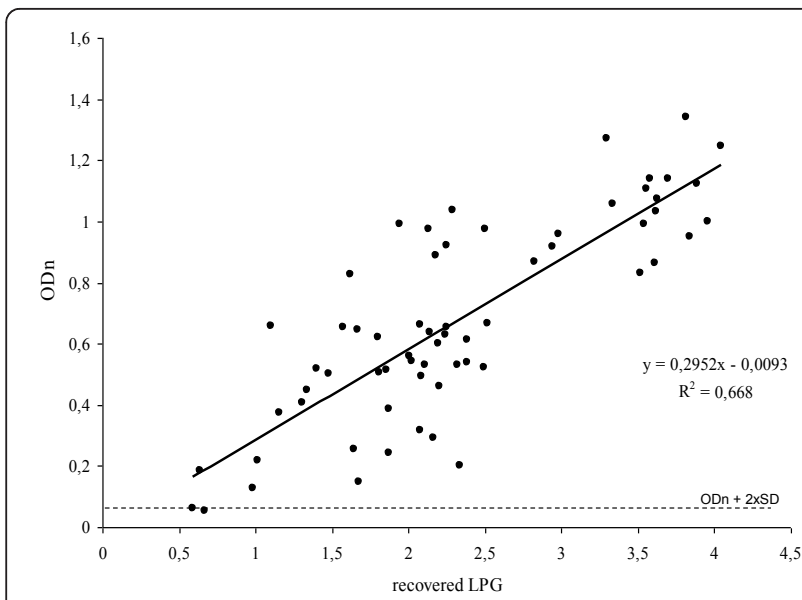

Figure 6 Relation between antibody level and recovered LPG Individual $\mathrm{OD}_{\mathrm{n}}$ values were plotted against ${ }^{10} \mathrm{log}$ transformed LPG, calculated from the number of larvae per rat that was isolated by artificial digestion. Data points represent individual animals of both the low- and high dose infection experiments. All but one point are above the cut-off level $\left(O D_{n}\right.$ 0.061).

with $16000 \mathrm{ML}$, although some ML could be identified in the diaphragm after histology. This might indicate that the intramuscular larvae might have been destroyed after digestion at this early developmental stage, since the protective nurse cell formation process starts at 8 dpi and is completed only on day 26 pi [17].

One of our goals was to determine whether ELISA was a suitable tool for detecting $T$. spiralis in rats. Therefore, it would be necessary to determine (1) the correlation between OD and infection dose in time and (2) the correlation between measured OD and exact numbers of $T$. spiralis ML in the rat. This is important

Table 1 Low estimate ( 0.05 percentile) and high estimate ( 0.95 percentile) of the conditional distribution of expected LPG given the OD value.

\begin{tabular}{crc}
\hline OD measured & Low estimate LPG & High estimate LPG \\
\hline 0 & 3 & 19 \\
0,1 & 5 & 31 \\
0,2 & 7 & 51 \\
0,3 & 12 & 84 \\
0,4 & 19 & 138 \\
0,5 & 31 & 228 \\
0,6 & 51 & 377 \\
0,7 & 84 & 623 \\
0,8 & 138 & 1029 \\
0,9 & 228 & 1700 \\
1 & 376 & 2809 \\
1,1 & 621 & 4642 \\
1,2 & 1025 & 7672 \\
1,3 & 1694 & 12678 \\
1,4 & 2799 & 20953 \\
\hline
\end{tabular}

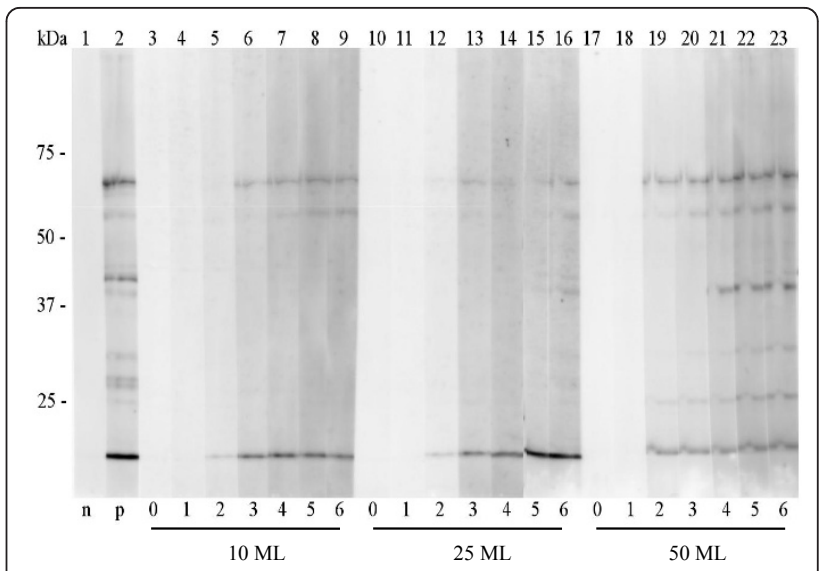

Figure 7 Immune response of experimentally $T$. spiralis infected rats as determined in Western blot. Lane 1: negative control (pooled pre-immune rat sera), lane 2: positive control (day 42 serum of a rat infected with $200 \mathrm{~T}$. spiralis ML), lanes 3-9: experimental rat serum 0-6 weeks pi with $10 \mathrm{ML}$ infection dose, lanes 10-16: experimental rat serum 0-6 weeks pi with $25 \mathrm{ML}$ infection dose, lanes 17-23: experimental rat serum 0-6 weeks pi with $50 \mathrm{ML}$ infection dose.

to be able to evaluate ELISA results of future serological surveys in wild rats.

There was a clear antibody response in all infection groups. Seroconversion in rats infected with $25 \mathrm{ML}$ or more, took place between days 7 and 14 pi, but already at day $7 \mathrm{pi}, \mathrm{OD}_{\mathrm{n}}$ levels of rats infected with 14 000-16 $000 \mathrm{ML}$ were above cut-off. Rats infected with $10 \mathrm{ML}$ showed a gradual linear increase in OD above the cutoff level from 14 to $42 \mathrm{dpi}$.

We found a positive correlation between $\mathrm{OD}_{\mathrm{n}}$ values and infection dose, although less pronounced in the 10$400 \mathrm{ML}$ infection groups. Some studies, also performed in rats, focused mainly on immune response with a limited number of infective doses. Salinas-Tobon et al. [18] performed experiments in rats with infection doses of 700, 2 000, 4000 and 8000 ML. Specific antibodies did not increase proportionally with the different ML doses tested, but the seroconversion period that occurred between 10 and $19 \mathrm{dpi}$, varied according to the infection dose.

We found a positive correlation between the number of recovered ML and serum antibody levels, although the predictive value of measured OD to estimate infection levels is limited, reflecting host variation in the immune response against a Trichinella infection.

The kinetics of anti-T. spiralis newborn larvae (NBL) immunity and its dose effects were studied in vivo by Wang [19]. In that study, rats were infected with 500, 2 000, 5000 and $6000 \mathrm{ML}$ and immune response was measured as a reduction in the numbers of recoverable NBL after intravenous challenge with 10 000-100 000 
NBL on day 16 pi. One of the results of that study was that infection with $2000 \mathrm{ML}$ per os induced the strongest immunity and that high dose immunization might induce a suppressive effect on host immunity. Our results demonstrate the exhausting effect on rats for doses above $8000 \mathrm{ML}$ and the more moderate clinical effects for doses of 6000 and $8000 \mathrm{ML}$. The lower immune effects with 5000 and $6000 \mathrm{ML}$ observed by Wang [19] could reflect this strong impact on rat health rather than a real suppressive effect on the immune system. Serum antibodies were not measured in that study.

In our study, infection dose and OD correlated linearly at all time points after infection with 2 000-10 000 T. spiralis ML. Infection with lower doses resulted in a positive correlation with OD, but only after $14 \mathrm{dpi}$ or more, and at a lower, though increasing level with time. Infection with higher doses (12 000 and $14000 \mathrm{ML}$ ) resulted in lower numbers of recovered larvae as compared to the $10000 \mathrm{ML}$ infection group (70\% and 60\% respectively), which explains the observed comparable decline in $\mathrm{OD}_{\mathrm{n}}$ between 28 and 42 dpi. However, at day 7 pi the $O D_{n}$ values in the $12000-16000 \mathrm{ML}$ infection groups are considerably higher than those of the 10000 ML group.

Rats that were infected with 10,25 or $50 \mathrm{ML}$ showed a serological response in the $T$. spiralis western blot 2 weeks post infection. Other rodents like mice have been experimentally infected with low doses of 50,10, and 5 ML of Trichinella spiralis per animal [20,21]. Seroconversion was observed in these experiments at $30 \mathrm{dpi}$ with an ES-ELISA and specific antibodies increased until 80 dpi. Measured splenic T-lymphocyte activity increased from day 10 to day $15 \mathrm{pi}$, even with $10 \mathrm{ML}$ as an infective dose [21]. This implies that for epidemiological studies, low infection levels can be detected by serology, both in rats and mice and we confirmed that low infection doses of $T$. spiralis larvae induce the production of specific antibodies at detectable levels in rats. These low doses reflect the infection level that we can find in the sylvatic cycle. Hurnikova and Dublinskyl [22] underlined that Trichinella (britovi, pseudospiralis and spiralis) infection in wild foxes is usually below 20 LPG and far less with wild boar. In The Netherlands, Trichinella britovi infection in wild foxes is even lower with LPG ranging from 0.04 to 0.71 [23]. For confirmation of rat sera from animals with low Trichinella infection levels or higher infection levels early in the time course of infection that exhibit OD values around the cut-off, we showed that western blots are a suitable instrument.

The results of our study confirmed previously conducted studies that were performed with very limited infection dose ranges in other host species like cattle [24], sheep [25,26], goats [27,28], horses [29-31], wild boars, pigs and foxes [32-34]. In these studies, the animals were experimentally infected with $T$. spiralis or other Trichinella species. As in our study, in most of these experiments, the time point of seroconversion and specific antibody titer were also dose dependent.

In summary, we show that rats, even infected with a low dose of 10 Trichinella ML, develop an immune response, which can be detected by use of serological assays and this immunological response is dose dependent up to an infection level of $10000 \mathrm{ML}$. This indicates that the Trichinella ES-ELISA and Western blot are useful instruments for the detection of the presence of $T$. spiralis in sentinel populations like wild rats, which easily cross sylvatic-domestic borders. We also show that antibody levels can not be used to calculate exact LPG values in rats due to high variation in infection rates.

\section{Ethical Issues}

Experimental infections in rats were conducted according to the Dutch laws applicable. The Central Animal Laboratory of the National Institute for Public Health and the Environment, the Netherlands, possesses a license under the Dutch 'Animal Experiments Act'. In accordance with Section 14 of this act, an officer has been appointed to supervise the welfare of laboratory animals.

\section{Acknowledgements}

The study was supported by the Network of Excellence "Med-Vet-Net" (WP TrichiMed) and the Inspectorate of Health Protection and Veterinary Public Health, Food and Consumer Product Safety Authority (WWA), the Netherlands. We thank Dirk Elberts and Jeroen Adema for animal handling and supplying animal materials during the rat experiments.

\section{Author details}

${ }^{1}$ Laboratory for Zoonoses and Environmental Microbiology, National Institute for Public Health and the Environment (RIVM), Bilthoven, The Netherlands. ${ }^{2}$ Anses, ENVA, UPEC, Laboratory for Animal Health, Joint Research Unit, Molecular Biology, Parasitic and Fungal Immunology (JRU BIPAR), MaisonsAlfort, France.

\section{Authors' contributions}

FFJF generated and analyzed parasitological and serological data, and wrote the first draft of the manuscript. MF and AG contributed to the generation of parasitological data. KT carried out the statistical analysis of the data. MAK and PWW carried out the pathological analysis-, and data interpretation. IV and PB contributed to the design and analysis of the studies including generation of parasitological data. JWBG conceived and designed the experiments, coordinated the study and contributed to the manuscript first draft. All authors read, approved and contributed to the final manuscript.

\section{Competing interests}

The authors declare that they have no competing interests.

Received: 6 June 2011 Accepted: 30 November 2011

Published: 30 November 2011

\section{References}

1. Pozio E, Hoberg E, La Rosa G, Zarlenga DS: Molecular taxonomy, phylogeny and biogeography of nematodes belonging to the Trichinella genus. Infect Genet Evol 2009, 9:606-616. 
2. Pozio E: Factors affecting the flow among domestic, synanthropic and sylvatic cycles of Trichinella. Vet Parasitol 2000, 93:241-262.

3. Pozio E, Rinaldi L, Marucci G, Musella V, Galati F, Cringoli G, Boireau P, La Rosa G: Hosts and habitats of Trichinella spiralis and Trichinella britovi in Europe. Int J Parasitol 2009, 39:71-79.

4. Schad GA, Duffy CH, Leiby DA, Murrell KD, Zirkle EW: Trichinella spiralis in an agricultural ecosystem: transmission under natural and experimentally modified on-farm conditions. J Parasitol 1987, 73:95-102.

5. Leiby DA, Duffy CH, Murrell KD, Schad GA: Trichinella spiralis in an agricultural ecosystem: transmission in the rat population. J Parasitol 1990, 76:360-364.

6. Stojcevic D, Zivicnjak T, Marinculic A, Marucci G, Andelko G, Brstilo M, Pavo L, Pozio E: The epidemiological investigation of Trichinella infection in brown rats (Rattus norvegicus) and domestic pigs in Croatia suggests that rats are not a reservoir at the farm level. J Parasitol 2004, 90:666-670.

7. Mikkonen T, Valkama J, Wihiman H, Sukura A: Spatial variation of Trichinella prevalence in rats in Finnish waste disposal sites. J Parasitol 2005, 91:210-213.

8. Neghina R: Trichinellosis, a Romanian never-ending story. An overview of traditions, culinary customs, and public health conditions. Foodborne Pathog Dis 2010, 7:999-1003.

9. Oivanen L, Mikkonen T, Sukura A: An outbreak of trichinellosis in farmed wild boar in Finland. APMIS 2000, 108:814-818.

10. Jovic S, Djordjevic M, Kulisic Z, Pavlovic S, Radenkovic B: Infectivity of Trichinella spiralis larvae in pork buried in the ground. Parasite 2001, 8: S213-215.

11. Takumi K, Franssen F, Fonville M, Grasset A, Vallee I, Boireau P, Teunis P, van der Giessen J: Within-host dynamics of Trichinella spiralis predict persistent parasite transmission in rat populations. Int J Parasitol 2010, 40:1317-1324.

12. European-Commission: Regulation EC No $2075 / 2005$ of the European Parliament and of the Council of 5 December 2005 laying down specific rules on official controls for Trichinella in meat. Off J EC L 2005, 338:60-82.

13. Gamble HR: Trichinella spiralis: immunization of mice using monoclonal antibody affinity-isolated antigens. Exp Parasitol 1985, 59:398-404.

14. Psaroulaki A, Antoniou M, Toumazos P, Mazeris A, loannou I, Chochlakis D, Christophi N, Loukaides P, Patsias A, Moschandrea I, Tselentis Y: Rats as indicators of the presence and dispersal of six zoonotic microbial agents in Cyprus, an island ecosystem: a seroepidemiological study. Trans R SoC Trop Med Hyg 2010, 104:733-739.

15. Auli M, Fernandez E: Characterization of functional and morphological changes in a rat model of colitis induced by Trichinella spiralis. Dig Dis Sci 2005, 50:1432-1443.

16. Khan WI, Collins SM: Immune-mediated alteration in gut physiology and its role in host defence in nematode infection. Parasite Immunol 2004, 26:319-326.

17. Despommier DD: How Does Trichinella spiralis Make Itself at Home? Parasitol Today 1998, 14:318-323.

18. Salinas-Tobon Mdel R, Navarrete-Leon A, Mendez-Loredo BE, EsquivelAguirre D, Martinez-Abrajan DM, Hernandez-Sanchez J: Trichinella spiralis: strong antibody response to a $49 \mathrm{kDa}$ newborn larva antigen in infected rats. Exp Parasitol 2007, 115:160-167.

19. Wang $\mathrm{CH}$ : Characterization of kinetics of anti-Trichinella spiralis newborn larvae immunity in rats. Front Biosci 1998, 3:a38-46.

20. Reiterova K, Antolova D, Hurnikova Z: Humoral immune response of mice infected with low doses of Trichinella spiralis muscle larvae. Vet Parasitol 2009, 159:232-235.

21. Dvoroznakova E, Hurnikova Z, Kolodziej-Sobocinska M: Development of cellular immune response of mice to infection with low doses of Trichinella spiralis, Trichinella britovi and Trichinella pseudospiralis larvae. Parasitol Res 2011, 108:169-176.

22. Hurnikova Z, Dubinsky P: Long-term survey on Trichinella prevalence in wildlife of Slovakia. Vet Parasitol 2009, 159:276-280.

23. van der Giessen JW, Rombout Y, Franchimont HJ, La Rosa G, Pozio E: Trichinella britovi in foxes in The Netherlands. J Parasitol 1998, 84:1065-1068.

24. Smith HJ, Snowdon KE, Finley GG, Laflamme LF: Pathogenesis and serodiagnosis of experimental Trichinella spiralis spiralis and Trichinella spiralis nativa infections in cattle. Can J Vet Res 1990, 54:355-359.
25. Tomasovicova O, Corba J, Havasiova K, Rybos M, Stefancikova A: Experimental Trichinella spiralis infection in sheep. Vet Parasitol 1991 40:119-126.

26. Pajersky A, Tomasovicova O, Kincekova J, Zubricky P, Koren J: Susceptibility and reactivity of sheep to Trichinella spiralis infection. Vet Med (Praha) 1996, 41:233-240

27. Reina D, Munoz-Ojeda MC, Serrano F, Molina JM, Navarrete I: Experimental trichinellosis in goats. Vet Parasitol 1996, 62:125-132.

28. Korinkova K, Pavlickova Z, Kovarcik K, Koudela B: Distribution of muscle larvae and antibody dynamics in goats experimentally infected with Trichinella spiralis. Parasitol Res 2006, 99:643-647.

29. Smith HJ, Snowdon KE: Experimental Trichinella infections in ponies. Can J Vet Res 1987, 51:415-416.

30. Soule C, Dupouy-Camet J, Georges P, Fontaine JJ, Ancelle T, Delvigne A, Perret C, Collobert C: Biological and parasitic variations in horses infested and reinfested by Trichinella spiralis. Vet Res 1993, 24:21-31, (in French).

31. Pozio E, Sofronic-Milosavljevic L, Gomez Morales MA, Boireau P, Nockler K: Evaluation of ELISA and Western Blot Analysis using three antigens to detect anti-Trichinella IgG in horses. Vet Parasitol 2002, 108:163-178.

32. Moller LN, Petersen E, Gamble HR, Kapel CM: Comparison of two antigens for demonstration of Trichinella spp. antibodies in blood and muscle fluid of foxes, pigs and wild boars. Vet Parasitol 2005, 132:81-84.

33. Nockler K, Serrano FJ, Boireau P, Kapel CM, Pozio E: Experimental studies in pigs on Trichinella detection in different diagnostic matrices. Vet Parasitol 2005, 132:85-90

34. Korinkova K, Kovarcik K, Pavlickova Z, Svoboda M, Koudela B: Serological detection of Trichinella spiralis in swine by ELISA (enzyme-linked immunosorbent assay) using an excretory-secretory (E/S) antigen. Parasitol Res 2008, 102:1317-1320.

doi:10.1186/1297-9716-42-113

Cite this article as: Franssen et al:: Antibody response against Trichinella spiralis in experimentally infected rats is dose dependent. Veterinary Research 2011 42:113.

\section{Submit your next manuscript to BioMed Central and take full advantage of:}

- Convenient online submission

- Thorough peer review

- No space constraints or color figure charges

- Immediate publication on acceptance

- Inclusion in PubMed, CAS, Scopus and Google Scholar

- Research which is freely available for redistribution

Submit your manuscript at www.biomedcentral.com/submit
Ciomed Central 